\title{
Mutation pattern is an influential factor on functional mutation rates in cancer
}

\author{
Chuance Du ${ }^{1 *+}$, Xiaoyuan $\mathrm{Wu}^{2+}$ and Jia $\mathrm{Li}^{3}$
}

\begin{abstract}
Background: Mutation rates are consistently varied in cancer genome and play nimpor trole in tumorigenesis, however, little has been known about their function potential and impact on the tribution of functional mutations. In this study, we investigated genomic features which affect mutation pattern and th y viction importance of mutation pattern in cancer.

Methods: Somatic mutations of clear-cell renal cell carcinoma, liver canc 'any cuncer and melanoma and single nucleotide polymorphisms (SNPs) were intersected with 54 distinct genomic zures. Somatic mutation and SNP densities were then computed for each feature type. We constructe 1-Aib windows, in which each row (1-Mb window) contains somatic mutation, SNP densities and 54 feature vectors. Lorrelation analyses were conducted between somatic mutation, SNP densities and each feature vector. We $2 / 50$ built two random forest models, namely somatic mutation model (CSM) and SNP model to predis. atic mutation and SNP densities on a 1-Kb scale. The relation of CSM and SNP scores was further analyzed the tributions of deleterious coding variants predicted by SIFT and Mutation Assessor, non-coding functional rariar. vva uated with FunSeq 2 and GWAVA and disease-causing variants from HGMD and ClinVar databases.

Results: We observed a wide range of genc vic "ures which affect local mutation rates, such as replication time, transcription levels, histone marks and rec "tory ele, ents. Repressive histone marks, replication time and promoter contributed most to the CSM models, w ile, - ombination rate and chromatin organizations were most important for the SNP model. We showed low nutated reg, ons preferentially have higher densities of deleterious coding mutations, higher average scores of nor coding variants, higher fraction of functional regions and higher enrichment of disease-causing variants as compa to high mutated regions.
\end{abstract}

Conclusions: Somatic mutc ion densitues vary largely across cancer genome, mutation frequency is a major indication of function and influence Ory distribution of functional mutations in cancer.

Keywords: Clear-cel' al cell carcinoma, Liver cancer, Lung cancer, Melanoma, Mutation pattern, Random forest, Functional variants

\section{Backgrounc}

Cancer is a maligna, $A$ disease as the result of the accumulati of con atic mutations (base pair substitutions, insertions, lelecions, rearrangements and copy number anges and the disruption of functions of critiand pathways in normal cells. Over the past

*Correspondence: duchuance@126.com

${ }^{\dagger}$ Chuance Du and Xiaoyuan Wu contributed equally to this work

${ }^{1}$ Department of Urology, Ganzhou Hospital Affiliated to Nanchang

University, Ganzhou, Jiangxi province, China

Full list of author information is available at the end of the article
10 years, the rapid development and wide application of sequencing technology have enabled a full detection of somatic mutations in cancer genome. The big projects, such as The Cancer Genome Atlas and International Cancer Genome Consortium projects, have sequenced more than 25 thousand cancer genomes and exomes and provided tremendous mutation data, which facilitates a broad evaluation of mutation patterns and their roles in cancer initiation and development $[1,2]$. Studies have consistently shown that somatic mutation rates are not constant across cancer genome and a variety of genomic 
properties influence local mutation densities, for instance, mutation frequency is increased close to breakpoints of structural rearrangements [3]. Mutagenesis is also highly affected by genomic features such as replication timing $[4,5]$, transcription levels [6] and chromatin organizations [7] in various cancer types.

It's well accepted that somatic mutations play a pivotal role in carcinogenesis, however the extent to which mutation frequency affects cancer formation and development isn't completely understood. For example, tumor cells with enhanced mutation frequency are prone to accumulation of driver mutations that confer a growth advantage to tumor cells and therefore are likely to develop cancer [8]. Hypermutated cancer genome possesses the prevalent signatures in genes which are critical to cancer initiation and progression $[9,10]$. Moreover, recurrently mutated genes in a cohort of patients are regarded as cancer-driving genes under positive selection in cancers $[6,11]$. However, few studies have been conducted on the function potential of mutation spectrum and its relation with functional somatic mutations in cancer. In this study, we characterized mutation patterns of four cancer types, including ccRCC, liver cancer, lur s adenocarcinoma and melanoma. We observed a $w$ range of genomic features which affect local myation rates and showed the importance of mutation $f$ 'quे ncy with respect to functionalities of somatic mu tio Low mutated regions have higher densities c deleter as mutations, higher average scores of non-coding variants, higher fraction of functional regions, and higher enrichment of disease-causing variants from HGMD and ClinVar databases than high mutated regions, supnorting that mutation frequency is an important indicat or of function and exerts a great impact on the distrib tional mutations in cancer genome.

\section{Results}

\section{The somatic mutation profile ir cancer}

To investigate the mutatic patter h and what factors influence mutation rate concers, we obtained 71,424 somatic muto ion 14 paired ccRCC and normal samples $\left[\begin{array}{l}1 \\ 2,011,2\end{array}\right.$-variants from 25 melanoma patients [13], 15,976 from 24 lung adenocarcinoma patient d 881, o from 88 liver cancer patients [6] (see "N tho c" section). We also took $38,248,779$ SNPs (single "cleoude polymorphism) from the 1000 genomes projec pilot one [14] as a comparison. We intersecu natic mutations and SNPs with a wide range of genomic features, epigenetic properties and computec/the average mutation densities of somatic tations and SNPs for each feature type. Figure 1 and A litional file 1: Figure S1-S4 showed the somatic mutation and SNP densities associated to various features. We found functionally important regions, such as CDS, promoter, UTR and ncExons are relatively low mutated compared to introns of lncRNAs ( $P$ value $<0.05$ in most cases

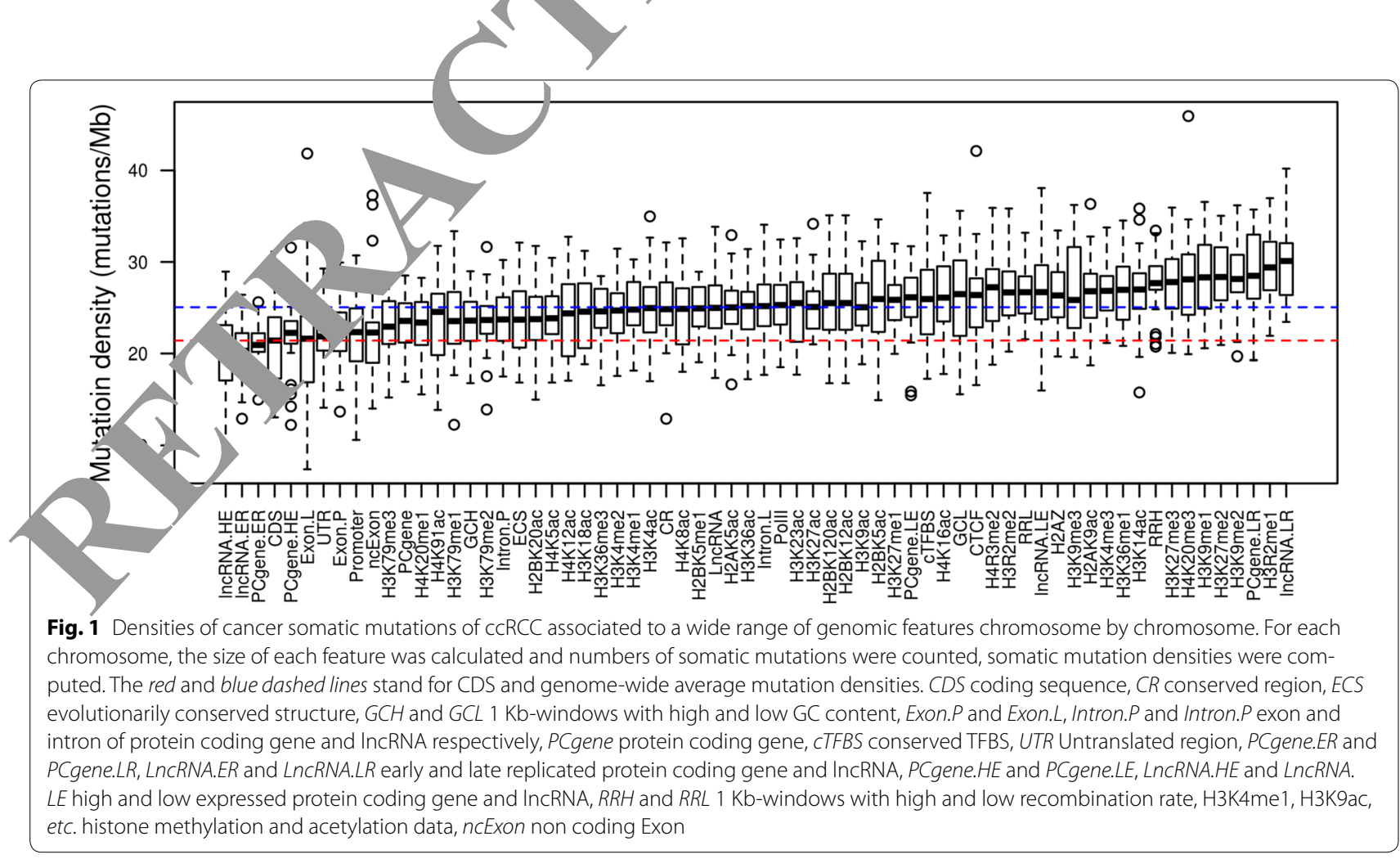


except for promoter in kidney cancer and ncExon in melanoma and CDS, UTR for SNPs, Wilcoxon rank sum test). Furthermore, we obtained 5 cancer driver genes in ccRCC, VHL, PBRM1, TCEB1, BAP1 and SETD2, from Sato's study [12], we found only two mutations, one in the CDS and another in the promoter of PBRM1, suggesting the CDS, promoters and UTR of these cancerdriving genes are mainly protected from mutations in ccRCC. Exons of either protein coding genes or lncRNAs showed lower somatic mutation rates relative to their introns respectively $(P$ value $<0.05$ for lncRNAs in all cases, $P$ value $<0.05$ for protein coding genes in liver and lung cancer, Wilcoxon rank sum test). However, no significant difference was observed on the SNP density between exons and introns of either protein coding genes or lncRNAs $(P$ value $>0.05$ in all cases, Wilcoxon rank sum test). Expression level and replication time are two important factors affecting cancer mutation and SNP rates, as evidenced by consistently lower mutation and SNP rates in high expressed and early replicated genes versus low expressed and late replicated ones $(P$ value $<0.05$ in all cases with a range from 0.008 to $2.429 \mathrm{e}-$ 13, Wilcoxon rank sum test). Repressive histone mark, such as H3K9me1, H3K9me2, H3K9me3, H3K27m and H3K27me3 are largely over-mutated in comr arison with active histone marks, such as H3K4me1, $\mathrm{H}$ K4 ne2, H3K79me1,H3K79me2,H3K79me3, and Hor 20 1 (P) values range from 0.3482 to $2.429 \mathrm{e}-13$ and are less than 0.05 in $95.83 \%$ of cases for cancer somatic mutations, $\mathrm{P}$ values range from 0.8618 to $3.5742 \mathrm{e}-09$ and are less than 0.05 in $73.33 \%$ of cases for SNPs, Wilcoxon rank sum test). Furthermore, in order to further characterize the relation between somatic mutation, $\mathrm{SN}$. and each feature, we constructed $28561-\mathrm{Mb}$, window in which each row (1-Mb window) contai somatic mutation, SNP densities and 54 feature recto (se "Methods" section). Correlation analyse were con acted between cancer somatic mutation, SN densitif $s$ and each feature vector. Features such as om replication time, CDS, UTR are most negat vely related with both somatic mutation and SNP lensities $=-0.3036$ to -0.6178 , $\mathrm{P}$ value $<1.5233 \mathrm{e} 61$ somatic mutations; $\mathrm{r}=-0.0264$ to -0.1219 , $\mathrm{P} \quad \mathrm{e}=1$. J48e-01-7.2937e-11 for SNPs). Repressive isto a marks, such as H3K9me1, H3K9me2, H3K9me3, n 20me3, H3K27me2 show high positive correltions wit soth somatic mutation and SNP densities $(\mathrm{r}=\mathrm{C}-0.6044$, P value $<1.1440 \mathrm{e}-12$ for somatic mutation ; $\mathrm{r}=0.3393-0.5178, \mathrm{P}$ value $=0$ for SNPs) (Fig. 2). 1, 1 general, SNP rates exhibit a large difference th somatic mutations, such as conserved regions and c1 BS (conserved transcription factor binding sites). Th. ese two regions are over-mutated in somatic mutations but protected from SNPs (Fig. 1). Recombination rates show strong positive correlation with SNPs but

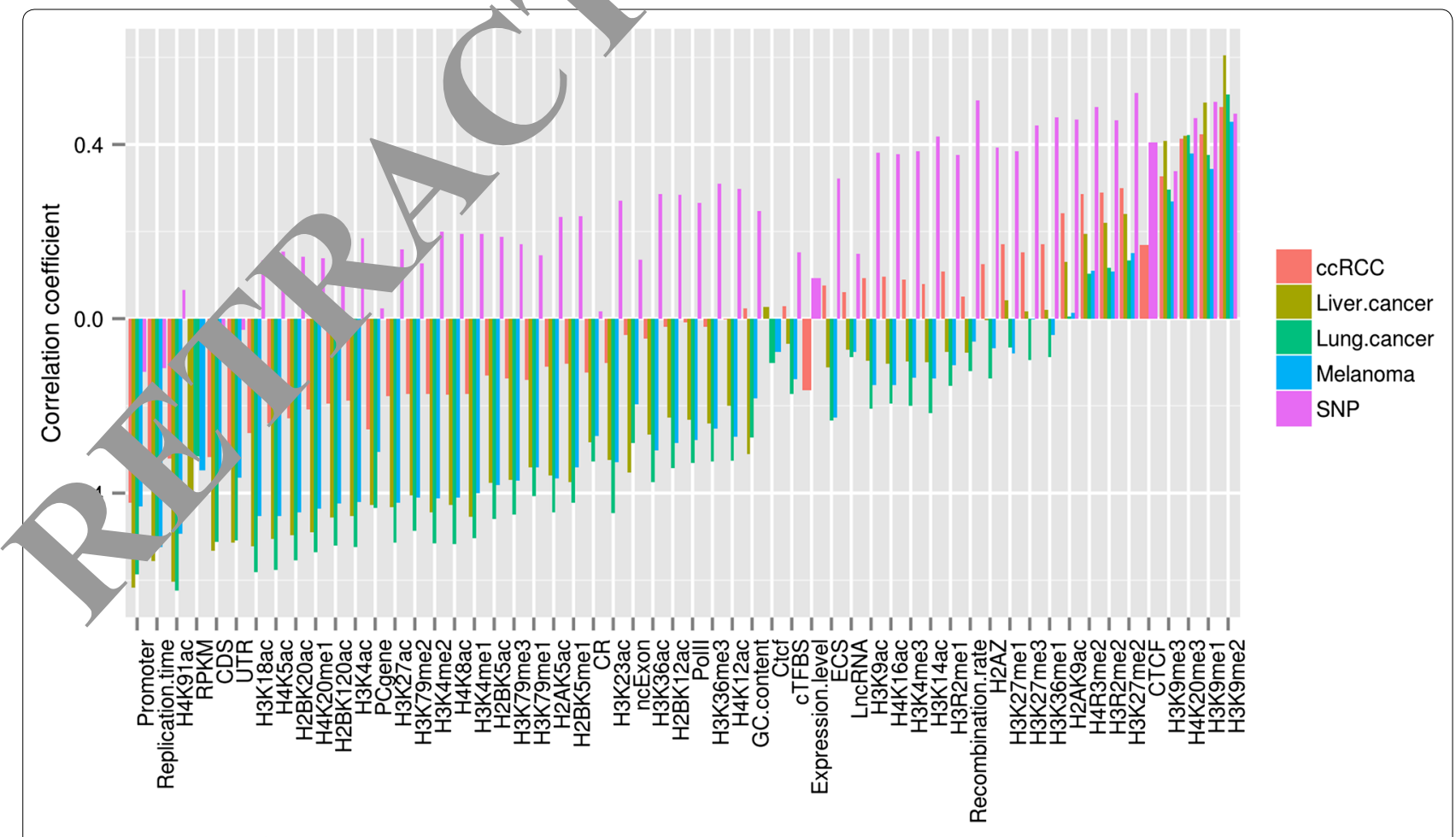

Fig. 2 Correlation coefficients of cancer somatic mutation (CSM), SNP densities with diverse genetic and epigenetic features at 1-Mb resolution 
a relatively weak correlation with somatic mutations $(\mathrm{r}=-0.1210-0.0499, \mathrm{P}$ value $<7.8871 \mathrm{e}-03$ for somatic mutations; $\mathrm{r}=0.5015$, $\mathrm{P}$ value $=0$ for SNPs). Moreover, multiple features have contrasting correlations with somatic mutation and SNP rates, such as H3K4ac, H4K5ac, H3K4me1, H3K4me2, H4K20me1 and ncExon (Fig. 2), suggesting distinct mutation patterns of somatic mutations and SNPs in cancer genome.

\section{Cancer somatic mutation (CSM) and SNP random forest models}

Given the sparse number of cancer mutations, we built two random forest models, referred to as the CSM and SNP models, with $28561-\mathrm{Mb}$ windows constructed above in order to analyze mutation distribution in more detail. \%IncMSE is computed from permuting a predictor's values over the original dataset, if a predictor is important in the model, the prediction error on the outof-bag portion of the data (MSE for regression) will be increased [15]. We used \%IncMSE to measure feature importance in the random Forest. In the CSM model, repressive histone marks such as $\mathrm{H} 3 \mathrm{~K} 9 \mathrm{me} 1, \mathrm{H} 3 \mathrm{~K} 9 \mathrm{me} 2$, H3K27me3, H3K27me2 and H4K20me3, replication tim. and promoter are among the most informative facto which corresponds to the findings in the correlatio. analyses (Fig. 3a; Additional file 1: Figure S5). How rer, features such as CDS, UTR have medium impor anc the CSM model, a possible explanation is the regions re relatively small in the genome and have litt mpact on genome-wide mutation rates, suppo-ung that 1 pressive histone marks, replication time an promoter contribute most to the CSM models. In the IP model, the most important features are recombinatio.. rate, H4R3me2, H4K20me3, ECS, H3R3me2 a line with the correlation analyst above (Fig. 3b).

\section{Functionality and mu ir..... uency}

Next, we asked whethe ytation frequencies are associated to fu ic ality and distribution of functional mutations In ordo examine this hypothesis, firstly, we con tructed annotation data which are overlapping $1-\mathrm{Mb} \mathrm{h}$ ows vith a step size $1 \mathrm{~Kb}$ using all 54 featu CSN d SNP densities (CSM and SNP scores) ere redicted with the CSM and SNP random models fo. ach $1 \mathrm{Mb}$-window and cancer type and averaged on a Kb scale (see "Methods" section). We applied SIFT and Mutation Assessor to predict damaging coding mutations for all cancer types. In total, 10,572, $1208,1625,2519$, and 7745, 1924,3009,3579 mutations were predicted as deleterious by SIFT and Mutation Assessor in ccRCC, liver cancer, lung cancer and melanoma respectively. We found markedly higher densities of deleterious mutations in the low CSM and SNP scoring regions as compared with those in the high CSM and SNP scoring ones. Moreover, negative correlations of average CSM and SNP scores were observed with densities of damaging mutations $(r=-0.6034$ to -0.9060 for CSM scores, $r=-0.4721$ for $\mathrm{N}$ P scores, $\mathrm{p}$ value $<0.05$ in all cases), suggesting stro o.tich nent of deleterious mutations in the low muta (Fig. 4a, b; Additional file 1: Figy S6). On the other hand, we computed functional s'ore $r$ all non-coding mutations with FunSeq 2 a d GWAV and analyzed their distributions with res 1 ct to mutation rates. We observed that hypomut ad i no with low CSM and SNP scores have high ar a age FunSeq 2 and GWAVA scores than hyperr tated or . Take the $200 \mathrm{Mb}$ intervals with the hig. est. lowest CSM and SNP scores as examples, the $\mathrm{Mb}$ iy erval with the lowest average CSM scorf has ioher average GWAVA and FunSeq 2 scores than $\mathrm{L}$. witn highest CSM score $(P$ value $<2.2 \mathrm{e}$ 16 in 11 cases, (coxon rank sum test); The differences of aver AVA and FunSeq 2 scores are relatively weak be+ween the $200 \mathrm{Mb}$ intervals with lowest SNP score anc/ highest SNP score $(\mathrm{P}$ value $=0.0090$ for WAVA, P value $=0.161$ for FunSeq 2, Wilcoxon rank su 1 test) (Fig. 4c, d; Additional file 1: Figure S7). Furthermore, we took $100 \mathrm{Mb}$ lowest and highest scoring CSM, SNP regions respectively and analyzed their distributions across various genetic features. As expected, functionally important regions, such as CDS, exons, UTR, splicing sites of protein coding genes and miRNAs, have 2-48-fold higher enrichment of low CSM and SNP scoring regions than high CSM and SNP scoring regions $(P$ value $<2.2 \mathrm{e}-16$ for all cases, $C$ hi squared test), however, nearly functionally neutral regions, such as lncRNAs and intron of lncRNAs are 1.5-2-fold more enriched with high CSM and SNP scoring regions relative to low CSM and SNP scoring ones $(P$ value $<2.2 \mathrm{e}-$ 16 for all cases, Chi squared test). Strikingly, fractions of low CSM and SNP scoring regions are 7 times higher than those of high CSM and SNP scoring regions in cancer-related miRNAs and genes ( $P$ value $<2.2 \mathrm{e}-16$ in all cases, Chi squared test) (Fig. 5a; Additional file 1: Figure S8). In addition, ccRCC driver genes, VHL, PBRM1, TCEB1, BAP1 and SETD2, are all located in the low scoring $1-\mathrm{Kb}$ windows, their average CSM scores are 21.436, 20.7088, 25.043, 20.7625 and 20.3447 respectively, which are less than the average (25.0582) in ccRCC. Lastly, we obtained non-coding disease-causing variants from HGMD and ClinVar databases and analyzed their distributions with regards to mutation rates. Disease-associated variants have lower average SNP and CSM scores than random SNPs ( $P$ value $<2.2 \mathrm{e}-16$ in all cases, Wilcoxon rank sum test) (Fig. 5b; Additional file 1: Figure S9). Low CSM and SNP scoring regions 


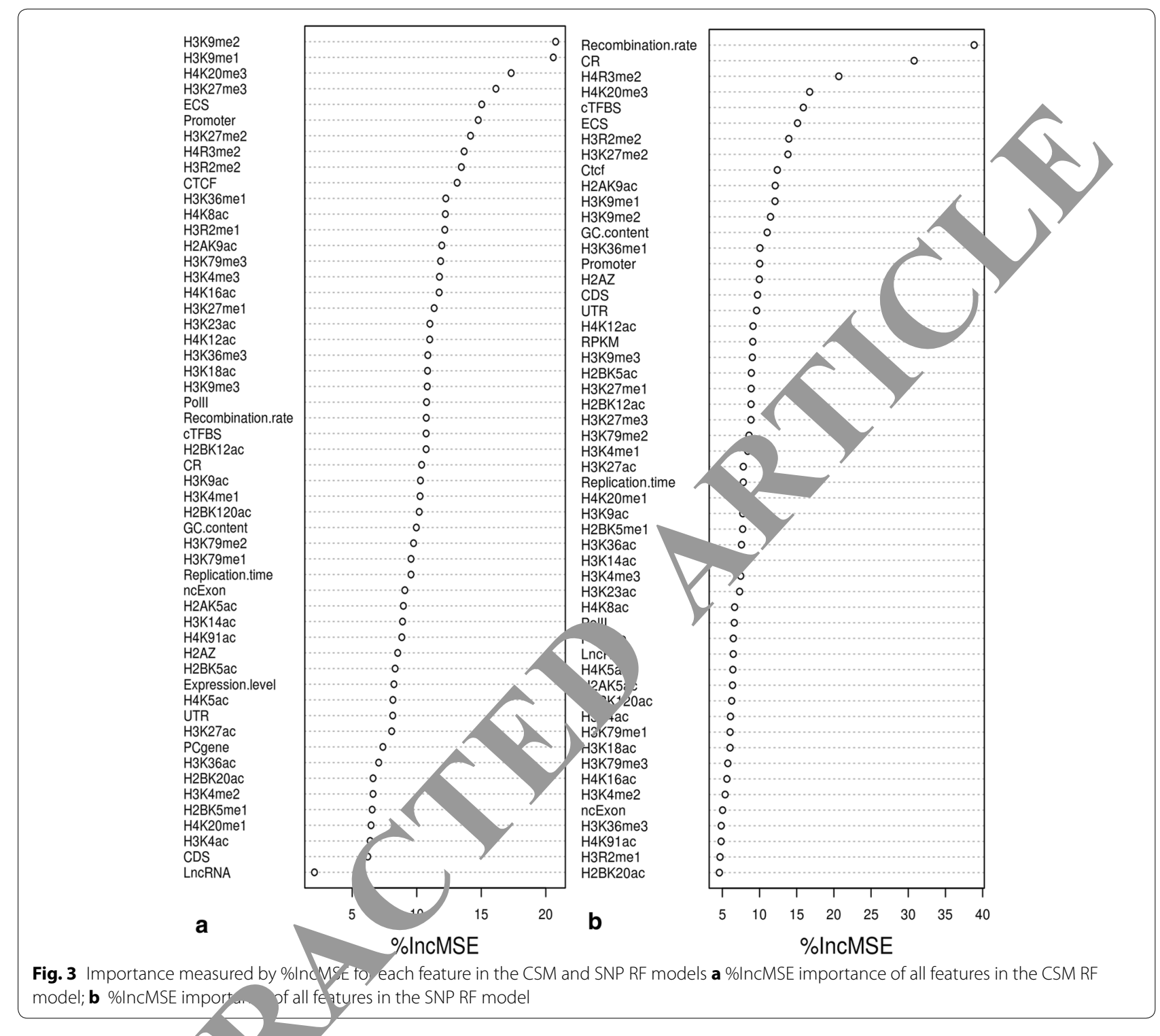

have higher vichmen of disease-causing variants than high C.M a SNP scoring ones. Take the lowest and higb est CSM ar, SNP scoring 200-Mb intervals as examp t e fo rmer has an 14-117 times higher average densi. of disease-causing variants than the latter (ए $v_{\text {c }}$ le <2. e-16 in all cases, Chi squared test). Siggative correlations were observed between ave CSM and SNP scores and densities of diseaseassociated variants $(\mathrm{r}=-0.6593$ to -0.7790 for CSM scores, -0.5718 for SNP scores, $\mathrm{P}$ values $<0.05$ in all cases) (Fig. 5c, d; Additional file 1: Figure S10). All these findings support the fact that mutation rate is an important indicator of function and an influential factor on the distribution of functional mutations in cancer genome.

\section{Discussion}

In this study, we have characterized the mutation spectrum in four cancer types and observed a wide range of genomic features that contribute to somatic mutation variations across cancer genome. The most influential features are replication time, transcription levels, repressive, active histone marks and regulatory elements. In line with many studies $[4-6,16]$, we found late replicated genes are more mutable as compared to early replicated ones, the mechanisms underlying this phenomenon might be explained in two ways. First of all, exhaustion of dNTP in the late stages of DNA replication might cause increase of single strand DNA regions which are more susceptible to mutagenesis $[17$, 18]; Secondly, mutation repair systems might erode 


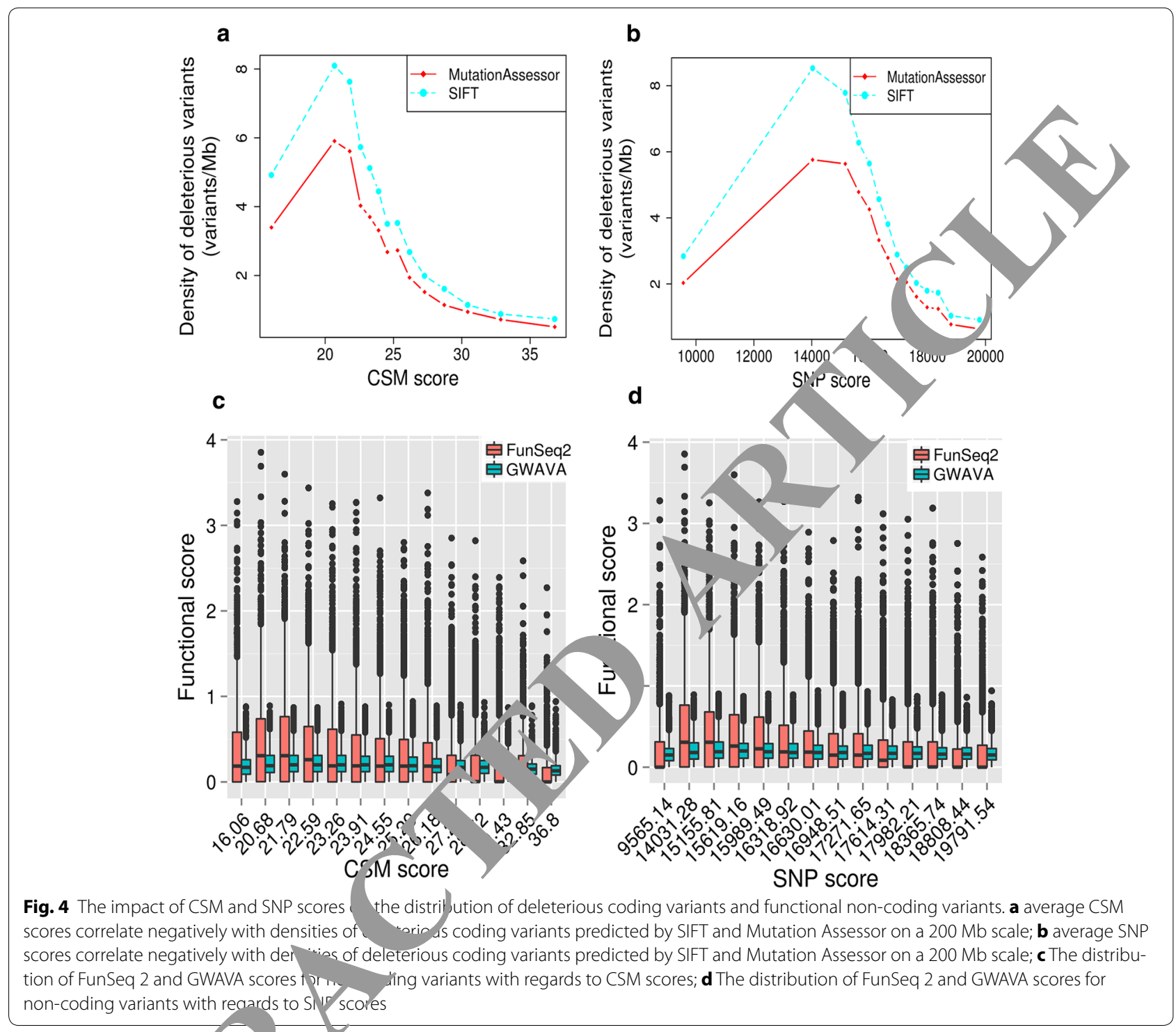

in the late rer ated gel $<$, leading to lack of efficient repair of mutatio 'esions [4]. Another feature associated with erevated $\mathrm{n}$, atation rate is low expressed genes. High th s iption might reduce number of mutations through nseription-coupled repair (TCR), which you repa more DNA lesions along with global pair (GGR) than GGR could operate alone in low niscribed regions [19]. TCR also in part accounts for mutation frequency variations among repressive, active histone marks, exons, CDS, UTR and introns. Regions, such as repressive condensed chromatin and introns, are subject to increased mutation rates, which could be due to more active TCR in highly transcribed open chromatin, CDS, UTR and exons. Regulatory elements like promoters show reduced local densities of somatic mutations, probably due to the integrity of nucleotide excision repair pathway consisting of global genome repair and TCR, which guarantees the efficient removal of mutation lesions [20]. Lastly, we found that recombination rates positively correlate with somatic mutation and SNP densities, which is in agreement with Lercher's study [21] but contrasts with that obtained by Renjamin's study [7]. In particular, recombination rates are a major influential factor on the SNP density, which is mostly attributed to mutagenesis of recombination rate and faulty repair of the double-strand breaks that initiate recombination [21]. Consistent with mutation pattern in cancers, we found that repressive histone marks, promoter and replication time contribute most to the CSM models, features like recombination rate 


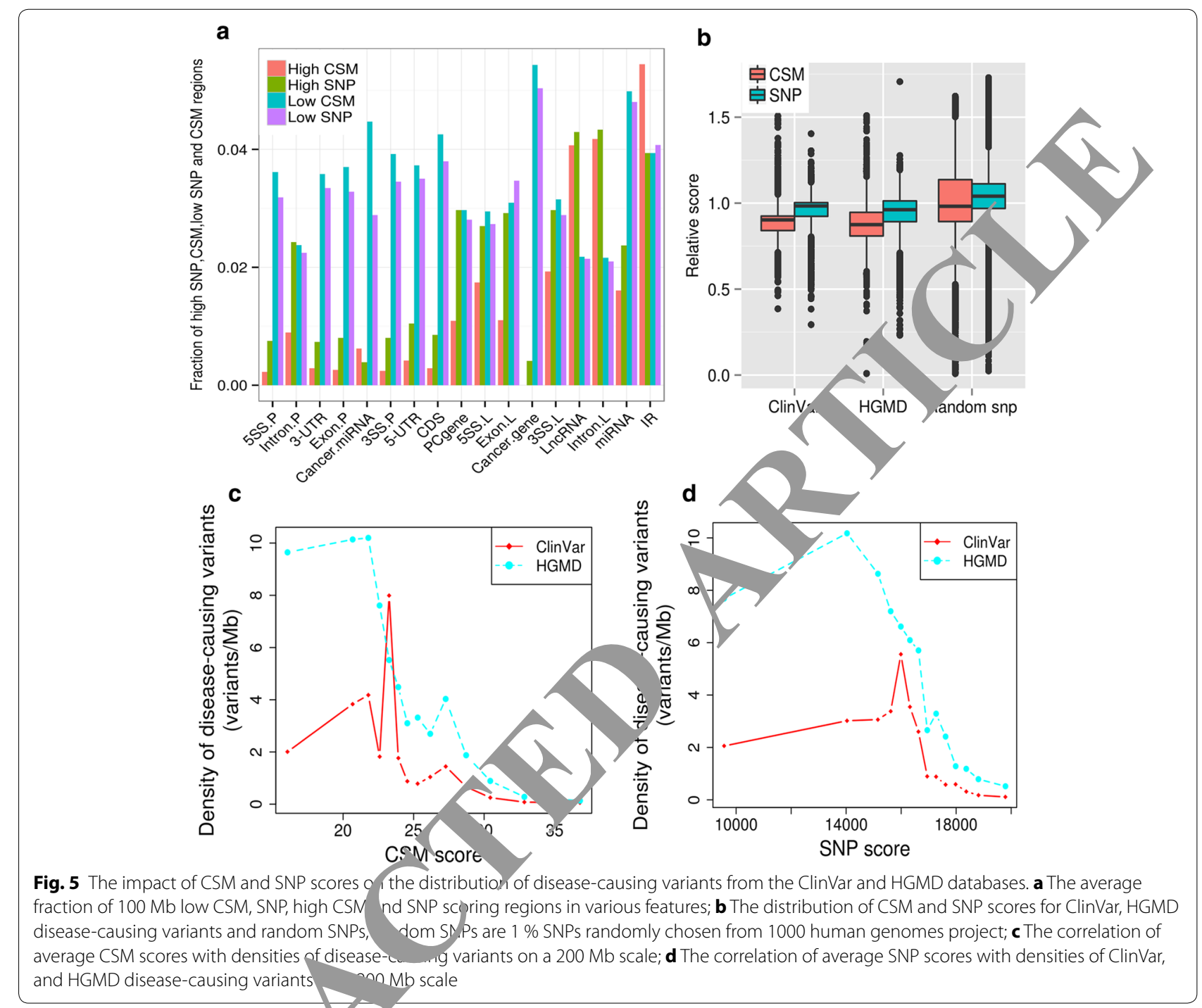

and chromatin o ganl. ions are most important for the SNP model.

Next, we a kea hether mutation rate variations are a byproduce of mutat $\mathrm{n}$ repair systems or represent cancer $m$, tio selection and function in cancer. Here we showed in tion frequencies are linked to the distribution func onal mutations in cancers. Low mutated $1 \mathrm{~d} d \mathrm{~d}$ to enrich functional mutations, including delc igus coding mutations, functional non-coding mutations and disease-causing mutations, suggesting their importance in the formation of functionalities of somatic mutations. Another evidence that further support this idea is low mutated regions possess strikingly higher enrichment of functional regions, such as CDS, exons, UTR, splicing sites of protein coding genes, cancer-related miRNAs and cancer driver genes as compared to high mutated regions, which explains why low mutated regions are prone to form functional mutations. Currently, many studies prefer to emphasize the importance of hypermutation in cancer initiation and development $[8,19]$, and hypermutation is an indicator of positive selection in cancer genes and multiple computational methods have been developed to detect them $[6,11,22]$. Our study show that hypomutated regions are mutation constraints and associated to functions in cancer genome, which should draw more attention and work in the future.

\section{Conclusions}

Taken together, somatic mutation densities vary largely across cancer genome, replication time, transcription levels, chromatin modifications and regulatory elements 
are among the features which most affect local mutation rates. To a large extent, mutation frequency is an indication of function and influence on the distribution of functional mutations in cancer.

\section{Methods \\ Mutation data}

Somatic variants were generated by whole genome sequencing of paired cancer and normal tissues and obtained from three studies: 2,011,261 variants from 25 melanoma patients [13], 1,845,976 variants from 24 lung adenocarcinoma patients, and 881,136 variants from 88 liver cancer patients [6] and 71,424 variants from 14 paired ccRCC and normal samples [12].Variants described as "substitution" or "indel" were both collected and are referred to collectively as mutations in the text. Germ line mutation data including 38,248,779 SNPs (single nucleotide polymorphism) were obtained from the 1000 genomes project [14] (http://www.1000genomes. org). Disease-associated variants data come from ClinVar (Version 2014/03/03, 55,689 variants) [23] and HGMD [24] (Version 2014/04/14, 166,768 variants) databases which are two common curations of variants related to human inherited diseases, coding variants were remov in this study, forming 6045 and 13,108 disease-imp. variants in the non-coding genome.

\section{Genome-wide data resources}

Human genome annotation were obta nea om Gencode V21, including protein coding g-nes, exons introns, UTR, non-coding exons (ncExon) ct [25]. Evolutionarily conserved bases with phast $\mathrm{Co}$ score greater than 117 were identified through alignmo... of 46 mammalian genomes with human $[26 \ldots+$ tionarily conserved structures (ECS) are RNA st condary structures predicted with a nouvel pip ine b sed on RNAz and SIS$\mathrm{SIz}$ in Smith MA's the Gerstein lab are reg tory regions $2.5 \mathrm{~Kb}$ from transcription sta $t$. (TSS) [28]. Genome-wide mapping of histone acetylat and methylation data of CD4+ T cell line were produced by ChIP-seq in Wang's [29] and Barski's ay [3] respectively, all coordinates conversic om $\mathrm{L}_{\mathrm{c}}$ assembly to hg19 was performed with the CSC I ift Over program [26]. Conserved TFBS (conse. d transcription factor binding sites, cTFBS) were gene, ed through aligning mouse and rat genomes with human [31]. Replication time data were obtained from Hepg2, Imr90, K562 and Bg02 cell lines for liver cancer, lung cancer, ccRCC and melanoma respectively (http:// genome.ucsc.edu/) ENCODE, 'Repli-seq track' [31]. Genome-wide replication timing was mapped to protein coding genes and lncRNAs, the $(\mathrm{G} 1 \mathrm{~b}+\mathrm{S} 1) /(\mathrm{S} 4+\mathrm{G} 2)$ ratio, early-to-late ratio, was determined for each protein coding gene and lncRNA. Genes that have a ratio greater than 1 or less than 1 were defined as early or late replicated genes respectively. Recombination rates (RR) were obtained from International HapMap Proiect (http:// hapmap.ncbi.nlm.nih.gov/) and averaged o ser successive $1-\mathrm{Kb}$ windows in human genome [32]. that have an average $R R$ above 4.0 were rega $d$ as high $R R$ regions ( $R R H)$, while low $R R$ rions ate $1-K b$ windows with recombination rate loss $4,0.5$ (RRL). GC content denotes the percentas of $\mathrm{G}$ or $\mathrm{c}$ ucleotides per $1-\mathrm{Kb}$ window. $1-\mathrm{Kb}$ windows hat have greater than $50 \%$ or less than $30 \% \mathrm{GC}$ vera are considered as high $(\mathrm{GCH})$ or low $\mathrm{GC}$ reg ins $\mathrm{CL})$ respectively [26].

RNA-seq data i cra forn / generated by sequencing 6 Hek293T ell s were downloaded from NCBI (GSE55572) [or exp, ssion analysis in ccRCC. Read alignment १s inducted with TopHat2 release 2.0.13 [34]. As for er cancer types, RNA-seq data in bam format were ac4 red from Hepg2, A549 and Nhek cell lines for. ancer, lung cancer and melanoma respectively [3\%. Read coverage was determined with bedtools v2.22.1 fo, lncRNAs and protein coding genes [35]. The mber of reads per Kilobase per million reads (RPKM) w. computed and averaged from three cell samples for each protein coding gene and lncRNA. Genes whose RPKM $>20$ or $<0.25$ were defined as high and low expressed respectively.

Cancer micro RNAs are a manual curation of mammalian miRNAs that have been experimentally characterized and actively involved in various cancers [36]. Cancer census genes are 547 cancer-driving genes annotated in COSMIC v71 (catalogue of somatic mutations in cancer) [37].

\section{Construction of 1-Mb windows and correlation analyses}

We used $1-\mathrm{Mb}$ window strategy to construct $1-\mathrm{Mb}$ windows for correlation analyses between SNP, cancer somatic mutation densities and genetic features as well as fitting Random forest models. Non-overlapping 1-Mb windows were formed across human genome, cancer mutations and SNPs were mapped into them and the number of somatic mutations and SNPs were counted for each $1-\mathrm{Mb}$ window. Genome-wide replication timing was mapped into $1-\mathrm{Mb}$ windows and the $(\mathrm{G} 1 \mathrm{~b}+\mathrm{S} 1) /$ $(\mathrm{S} 4+\mathrm{G} 2)$ ratio was computed for each $1-\mathrm{Mb}$ window. Read coverage was determined with bedtools v2.22.1 for each $1-\mathrm{Mb}$ window, exons from Gencode V21 were intersected with $1-\mathrm{Mb}$ windows and the length of exons was then calculated for each $1-\mathrm{Mb}$ window. The number of reads per Kilobase per million reads (RPKM) was computed and averaged from three cell samples for each $1-\mathrm{Mb}$ window. Recombination rates (RR) were averaged over successive $1-\mathrm{Mb}$ windows in the human genome. 
Regarding other features, the number of bases covered by each feature was calculated for each $1-\mathrm{Mb}$ window. As a number of $1-\mathrm{Mb}$ windows are lack of coverage of features and mutation information, partial $1-\mathrm{Mb}$ windows were discarded, including $1-\mathrm{Mb}$ windows defined as telomere, centromere, stalk, pericentromere, 1-Mb windows which are all undefined bases. The whole chromosome $\mathrm{Y}$ was ruled out from this study too, due to its consistently low mutation rates caused by gender bias. In total, $224.3 \mathrm{Mb}$ regions were abandoned in this study, forming 2856 $1-\mathrm{Mb}$ windows and 56 columns corresponding to cancer somatic mutation density, SNP density and 54 features ranging from conserved regions, promoters to histone modifications. Correlation analyses between SNP, cancer somatic mutation densities and each feature were performed in R.

\section{Random forest model}

The SNP and cancer somatic mutation (CSM) random forest (RF) models were constructed with the $\mathrm{R}$ random Forest package [15]. In the RF models, we used 2856 $1-\mathrm{Mb}$ windows constructed above and cancer mutation density (CSM model), SNP density (SNP model) response variables and 54 genomic features as predic. variables to build two RF models, CSM and SNP models. All predictor values were plus one and $\log$ rale to reduce the great variation of vectors. The num ber rees was set to 500, mtry was set to 20 for CSM nodel an 15 for SNP model, all other parameters wre s. o default values. Model calibration and validacion were y scribed in the Additional file 2.

For CSM and SNP score predict we used the same $1-\mathrm{Mb}$ window strategy as in the moder wulding, however, the $1 \mathrm{Mb}$-window was slided an the human genome with a step size of $1 \mathrm{~Kb}-1 \mathrm{Mb}$ indows overlapping telomere, centromere, sta or éricentromere regions, $1 \mathrm{Mb}$ windows whic $r$ ndefined bases and chromosome $\mathrm{Y}$ we remo $\mathrm{d}$ /from the annotation data, resulting in 2, $0 \quad 687 \mathrm{row}$ annotations. The CSM and SNP score were p. 'icted using the two RF models for each $1 \mathrm{Ab}$ window and averaged on a $1-\mathrm{Kb}$ window scale.

Co ption yses between CSM, SNP scores

\section{ad d loterious coding mutations, GWAVA and FunSeq 2 \\ nc roding scores, disease-causing variants}

Codi o mutations mainly came from two sources for ccRCC: whole genome sequencing of 14 paired ccRCC samples and exome sequencing of 325 paired ccRCC samples from TCGA [38]. The coding mutations of other cancer types were obtained from the same sources as described in the section of "Mutation data". Their functional impacts were predicted by SIFT [39], and Mutation assessor [40] respectively, variants were regarded as deleterious based on the following criteria: SIFT score $<0.05$, and Mutation Assessor socre >1.9. 70,659 ccRCC, 881,130 liver cancer, 1,623,242 lung cancer and 2,011,261 melanoma non-coding variants were scored with FunSeq 2 [41] (http://funseq2.gersteinlab.org/, n G GVAVA [42] (https://www.sanger.ac.uk/sanger/Statc Gwava) respectively, all the parameters we set to default. Deleterious coding mutations, no-co o v riants with GWAVA and FunSeq 2 score, and disea -causing variants from HGMD and Clin $r$ datab ises were mapped into $1-\mathrm{Kb}$ windows whi ha $\mathrm{h}$ age CSM and SNP scores. $1-\mathrm{Kb}$ window $\mathrm{we}$. then sorted based on SNP and CSM scores a dividea ito non-overlapping 200$\mathrm{Mb}$ intervals res ect $\mathrm{v}$. For each $200-\mathrm{Mb}$ interval, the following val were omputed, including the average densit; of teleterious coding mutations, diseasecausing varia average GWAVA and FunSeq 2 scores, averag CSM añ SNP scores. Correlation analyses were conducten between densities of deleterious coding mutation o, disease-causing variants and average CSM and SNP, cores.

\section{St istical analyses}

Dita were presented as mean, statistical differences between groups were computed with the Chi squared test (chisq.test) or Wilcoxon rank sum test (wilcox. test), correlation analysis (cor.test) was conducted in $\mathrm{R}$, $P<0.05$ was regarded statistically significant and the null hypothesis was rejected.

\section{Additional files}

Additional file 1. Supplementary figures.

Additional file 2. Calibration and validation of random forest models.

\section{Authors' contributions}

$J L$ was in charge of building random forest models, model validation, prediction of deleterious coding mutations with SIFT and Mutation assessor and scoring of noncoding variants with FunSeq 2 and GWAVA. Chuance Du analyzed and interpreted the results, Xiaoyuan WU collected cancer mutations and genomic features from different sources. CD and $J L$ drafted the manuscript. All authors read and approved the final manuscript.

\section{Author details}

${ }^{1}$ Department of Urology, Ganzhou Hospital Affiliated to Nanchang University, Ganzhou, Jiangxi province, China. ${ }^{2}$ Department of Rehabilitation, Ganzhou Hospital Affiliated to Nanchang University, Nan Chang, Jiangxi province, China. ${ }^{3}$ Department of Thyroid and Breast, Shanghai Tenth People's Hospital, Tongji University, Shanghai, 200072, China.

\section{Competing interests}

The authors declare that they have no competing interests.

Received: 11 June 2015 Accepted: 3 February 2016

Published online: 09 February 2016 


\section{References}

1. Hudson TJ, Anderson W, Artez A, Barker AD, Bell C, Bernabé RR, Bhan MK, Calvo F, Eerola I, Gerhard DS, Guttmacher A, Guyer M, Hemsley FM, Jennings JL, Kerr D, Klatt P, Kolar P, Kusada J, Lane DP, Laplace F, Youyong L, Nettekoven G, Ozenberger B, Peterson J, Rao TS, Remacle J, Schafer AJ, Shibata T, Stratton MR, Vockley JG, et al. International network of cancer genome projects. Nature. 2010;464:993-8.

2. Chin L, Andersen JN, Futreal PA. Cancer genomics: from discovery science to personalized medicine. Nat Med. 2011;17:297-303.

3. Drier Y, Lawrence MS, Carter SL, Stewart C, Gabriel SB, Lander ES, Meyerson M, Beroukhim R, Getz G. Somatic rearrangements across cancer reveal classes of samples with distinct patterns of DNA breakage and rearrangement-induced hypermutability. Genome Res. 2013;23:228-35

\section{(20121108-genome_research)}

4. Stamatoyannopoulos JA, Adzhubei I, Thurman RE, Kryukov GV, Mirkin SM, Sunyaev SR. Human mutation rate associated with DNA replication timing. Nat Genet. 2009;41:393-5.

5. Woo YH, Li W-H. DNA replication timing and selection shape the landscape of nucleotide variation in cancer genomes. Nat Commun. 1004;2012:3.

6. Lawrence MS, Stojanov P, Polak P, Kryukov GV, Cibulskis K, Sivachenko A, Carter SL, Stewart C, Mermel CH, Roberts SA, Kiezun A, Hammerman PS, McKenna A, Drier Y, Zou L, Ramos AH, Pugh TJ, Stransky N, Helman E, Kim J, Sougnez C, Ambrogio L, Nickerson E, Shefler E, Cortés ML, Auclair D, Saksena G, Voet D, Noble M, DiCara D, et al. Mutational heterogeneity in cancer and the search for new cancer-associated genes. Nature. 2013:499:214-8.

7. Schuster-Böckler B, Lehner B. Chromatin organization is a major influence on regional mutation rates in human cancer cells. Nature. 2012;488:504-7

8. Fox EJ, Prindle MJ, Loeb LA. Do mutator mutations fuel tumorigenesis? Cancer Metastasis Rev. 2013;32:353-61.

9. Burns MB, Temiz NA, Harris RS. Evidence for APOBEC3B mutagen in multiple human cancers. Nat Genet. 2013;45:977-83.

10. Hoang ML, Chen CH, Sidorenko VS, He J, Dickman KG, Yun B , riv M, Niknafs N, Douville C, Karchin R, Turesky RJ, Pu YS, Vogels:.t. B, R. topoulos N, Grollman AP, Kinzler KW, Rosenquist TA. Mu+ onal signat of aristolochic acid exposure as revealed by whole-ey me uencing $\mathrm{NIIH}$ Public Access. Sci Transl Med. 2013;5:197ra102.

11. Dees ND, Zhang Q, Kandoth C, Wendl MC, Schie aing W, Koboldt C, Mooney TB, Callaway MB, Dooling D, Mardis ER, Wilson K, Ding L. MuSiC: identifying mutational significance in cancer genomes. C ome Res. 2 12;22:1589-98.

12. Sato $Y$, Yoshizato $T$, Shiraishi $Y$, Maekawa $S, O$ Y Karnura T, Shimamura T, Sato-Otsubo A, Nagae G, Suzuki W' Nagata Y, Yusnida K, Kon A, Suzuki Y, Chiba K, Tanaka H, Niida A, Fujimo noda T, Morikawa T, Maeda D, Kume H, Sugano S, Fukayama M, A uratarn A, Sanada M, Miyano S, Homma Y, Ogawa S. Intea molecu ar analysis of clear-cell renal cell carcinoma. Nat Genet. 013;4, 60-7.

13. Berger MF, Hodis $E_{1}$ Hen ap Je YL, Lawrence MS, Protopopov A Ivanova E, Watsor in, Nickt E, Ghosh P, Zhang H, Zeid R, Ren X, Cibulskis K, Sivacher AY, Wagle $\$ acker A, Sougnez C, Onofrio R, Ambrogio L, Auclair D, enn Carter SL, Drier Y, Stojanov P, Singer MA, Voet D, Jing $R$, Saksen $G$, Barretiı $s t / a l$. Melanoma genome sequencing reveals freque it PREX2 mutatio, ns. Nature. 2012;485:502-6.

14. Clar Zf ong-Bradley X, Smith R, Kulesha E, Xiao C, Toneva I, Vaughan B, Preuss sinone $R$, Shumway M, Sherry S, Flicek P. The 1000 Genomes iect: da. nagement and community access. Nat Methods. $202 ; 9: 459-62$.

Random forests. Mach Learn. 2001;45:5-32.

16. $L$, De $S$, Michor F. DNA replication timing and higher-order nuclear Or hization determine single-nucleotide substitution patterns in cancer genomes. Nat Commun. 2013;4:1502.

17. Mirkin EV, Mirkin SM. Replication fork stalling at natural impediments. Microbiol Mol Biol Rev. 2007:71:13-35.

18. Yang Y, Sterling J, Storici F, Resnick MA, Gordenin DA. Hypermutability of damaged single-strand DNA formed at double-strand breaks and uncapped telomeres in yeast Saccharomyces cerevisiae. PLoS Genet 2008;4(11):e1000264.

19. Roberts SA, Gordenin DA. Hypermutation in human cancer genomes: footprints and mechanisms. Nat Rev Cancer. 2014;14(12):786-800.
20. Polak P, Lawrence MS, Haugen E, Stoletzki N, Stojanov P, Thurman RE, Garraway LA, Mirkin S, Getz G, Stamatoyannopoulos JA, Sunyaev SR. Reduced local mutation density in regulatory DNA of cancer genomes is linked to DNA repair. Nat Biotechnol. 2014;32:71-5.

21. Lercher MJ, Hurst LD. Human SNP variability and mutation rate are higher in regions of high recombination. Trends Genet. 2002;18.33\%-40.

22. Lawrence MS, Stojanov P, Mermel CH, Robinson JT, G rawo $L A$, Golub TR, Meyerson M, Gabriel SB, Lander ES, GetzG. 'sery ar saturation analysis of cancer genes across 21 tumour typu atı 2014;505:495-501

23. Landrum MJ, Lee JM, Riley GR, Jang W, Rublin in WS, Ch rch DM, Maglott DR. ClinVar: public archive of rel tionsh, amo sequence variation and human phenotype. Nucle c Acids Res. 20 ,2014(42):980-5.

24. Stenson PD, Mort M, Ball EV, Howel K, Phillips AD, Thomas NS, Cooper DN. The Human Gene Mutation Da ase: 2008 update. Genome Med. 2009;1:13.

25. Harrow J, Frankish A, Gorralež Tapanari E, Diekhans M, Kokocinski F, Aken BL, Barrell D, Z? dissa A, Sea. Barnes I, Bignell A, Boychenko V, Hunt T, Kay M, My ki G, Rajan,, Despacio-Reyes G, Saunders G, Steward C, Harte $K_{,}$Lin $M_{1}$, wald C, Tanzer A, Derrien T, Chrast J, Walters N, Balasubram i S, Pei B, os M, et al. GENCODE: the reference human ger ome c hotation for the ENCODE project. Genome Res. 2012;22:i

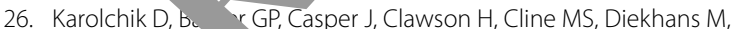
Dreszer TR, Fujità Guruvadoo L, Haeussler M, Harte RA, Heitner S, Hini Learned K, Lee BT, Li CH, Raney BJ, Rhead B, Rosenbloom KR, Sl an Cr, peir ML, Zweig AS, Haussler D, Kuhn RM, Kent WJ. The UCSC enome Browser database: 2014 update. Nucleic Acids Res. 2014;42 $764-70$.

Smith MA, Gesell T, Stadler PF, Mattick JS. Widespread purifying selection on RNA structure in mammals. Nucleic Acids Res. 2013;41:8220-36.

8. Khurana E, Fu Y, Colonna V, Mu XJ, Kang HM, Lappalainen T, Sboner A, Lochovsky L, Chen J, Harmanci A, Das J, Abyzov A, Balasubramanian S, Beal K, Chakravarty D, Challis D, Chen Y, Clarke D, Clarke L, Cunningham F, Evani US, Flicek P, Fragoza R, Garrison E, Gibbs R, Gümüs ZH, Herrero J, Kitabayashi N, Kong Y, Lage K, et al. Integrative annotation of variants from 1092 humans: application to cancer genomics. Science. 2013;342:1235587.

29. Wang Z, Zang C, Rosenfeld JA, Schones DE, Barski A, Cuddapah S, Cui K, Roh T-Y, Peng W, Zhang MQ, Zhao K. Combinatorial patterns of histone acetylations and methylations in the human genome. Nat Genet. 2008:40:897-903.

30. Barski A, Cuddapah S, Cui K, Roh TY, Schones DE, Wang Z, Wei G, Chepelev I, Zhao K. High-Resolution Profiling of Histone Methylations in the Human Genome. Cell. 2007;129:823-37.

31. Rosenbloom KR, Sloan CA, Malladi VS, Dreszer TR, Learned K, Kirkup VM, Wong MC, Maddren M, Fang R, Heitner SG, Lee BT, Barber GP, Harte RA Diekhans M, Long JC, Wilder SP, Zweig AS, Karolchik D, Kuhn RM, Haussler D, Kent WJ. ENCODE Data in the UCSC Genome Browser: year 5 update. Nucleic Acids Res. 2013;41:56-63.

32. Altshuler DM, Gibbs RA, Peltonen L, Altshuler DM, Gibbs RA, Peltonen L, Dermitzakis E, Schaffner SF, Yu F, Peltonen L, Dermitzakis E, Bonnen PE, Altshuler DM, Gibbs RA, de Bakker PIW, Deloukas P, Gabriel SB, Gwilliam R, Hunt S, Inouye M, Jia X, Palotie A, Parkin M, Whittaker P, Yu F, Chang K, Hawes A, Lewis LR, Ren $Y$, Wheeler D, et al. Integrating common and rare genetic variation in diverse human populations. Nature. 2010;467:52-8.

33. Schwartz S, Mumbach MR, Jovanovic M, Wang T, Maciag K, Bushkin GG, Mertins P, Ter-Ovanesyan D, Habib N, Cacchiarelli D, Sanjana NE, Freinkman E, Pacold ME, Satija R, Mikkelsen TS, Hacohen N, Zhang F, Carr SA, Lander ES, Regev A. Perturbation of m6A writers reveals two distinct classes of mRNA methylation at internal and $5^{\prime}$ sites. Cell Rep. 2014:8:284-96.

34. Kim D, Pertea G, Trapnell C, Pimentel H, Kelley R, Salzberg SL. TopHat2: accurate alignment of transcriptomes in the presence of insertions, deletions and gene fusions. Genome Biol. 2013;14:R36.

35. Quinlan AR, Hall IM. BEDTools: a flexible suite of utilities for comparing genomic features. Bioinformatics. 2010;26:841-2.

36. Xie B, Ding Q, Han H, Wu D. MiRCancer: a microRNA-cancer association database constructed by text mining on literature. Bioinformatics. 2013;29:638-44 
37. Forbes SA, Bindal N, Bamford S, Cole C, Kok CY, Beare D, Jia M, Shepherd R, Leung K, Menzies A, Teague JW, Campbell PJ, Stratton MR, Futreal PA. COSMIC: mining complete cancer genomes in the catalogue of somatic mutations in cancer. Nucleic Acids Res. 2011;39(suppl. 1):945-50.

38. Chang K, Creighton CJ, Davis C, Donehower L, Drummond J, Wheeler D, Ally A, Balasundaram M, Birol I, Butterfield YSN, Chu A, Chuah E, Chun H-JE, Dhalla N, Guin R, Hirst M, Hirst C, Holt RA, Jones SJM, Lee D, Li HI, Marra MA, Mayo M, Moore RA, Mungall AJ, Robertson AG, Schein JE, Sipahimalani P, Tam A, Thiessen N, et al. The Cancer Genome Atlas Pan-Cancer analysis project. Nat Genet. 2013;45:1113-20.
39. Ng PC, Henikoff S. SIFT: predicting amino acid changes that affect protein function. Nucleic Acids Res. 2003;31:3812-4.

40. Reva B, Antipin Y, Sander C. Predicting the functional impact of protein mutations: application to cancer genomics. Nucleic Acids Res. 2011;39:37-43.

41. Fu Y, Liu Z, Lou S, Bedford J, Mu XJ, Yip KY, Khurana E, Ge stern M. FunSeq2: a framework for prioritizing noncoding rec ator, variants in cancer. Genome Biol. 2014;15:1-15.

42. Ritchie GRS, Dunham I, Zeggini E, Flicek P. Functional ann noncoding sequence variants. Nat Methods 0 014;11:294-6.

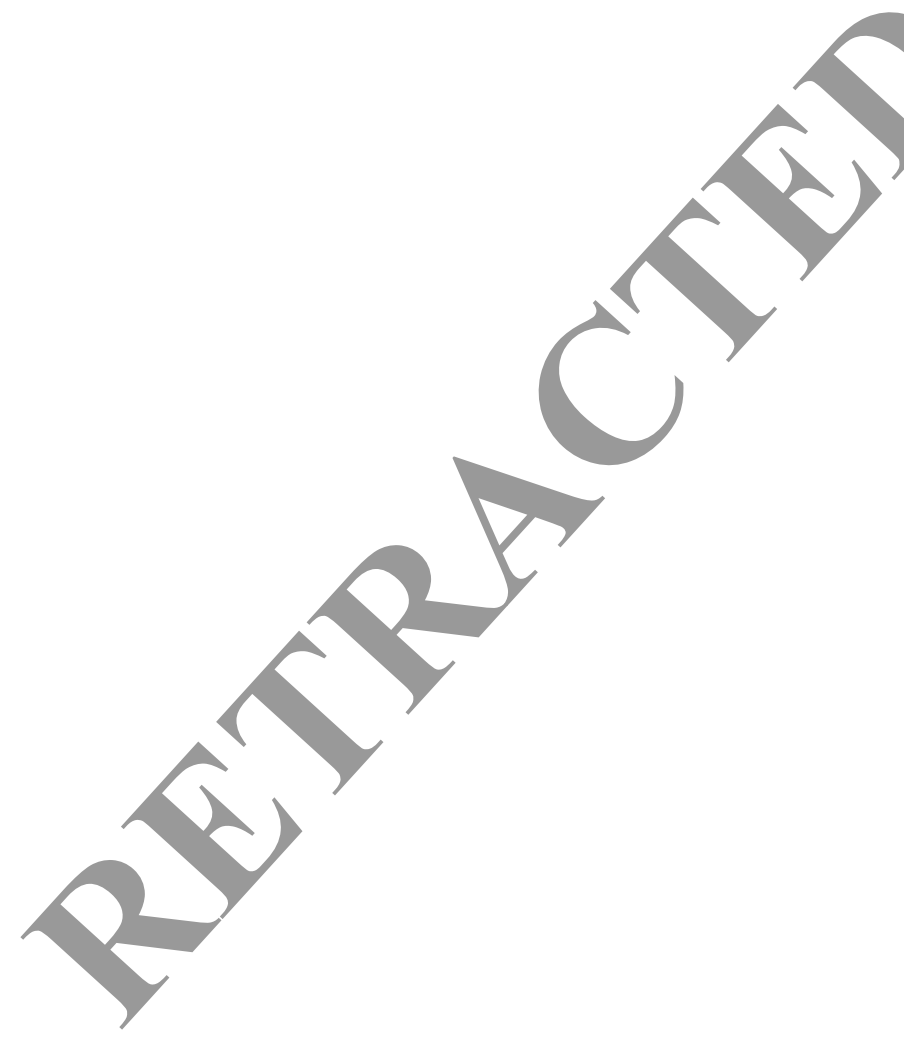

Submit your next manuscript to BioMed Central and we will help you at every step:

- We accept pre-submission inquiries

- Our selector tool helps you to find the most relevant journal

- We provide round the clock customer support

- Convenient online submission

- Thorough peer review

- Inclusion in PubMed and all major indexing services

- Maximum visibility for your research

Submit your manuscript at www.biomedcentral.com/submit

C) Biomed Central 